\title{
Integrability and regularity of the flow of stochastic differential equations with jumps
}

\author{
Jean-Christophe Breton* \\ Univ Rennes \\ CNRS, IRMAR - UMR 6625 \\ 263 Avenue du Général Leclerc \\ F-35000 Rennes, France
}

\author{
Nicolas Privault ${ }^{\dagger}$ \\ Division of Mathematical Sciences \\ School of Physical and Mathematical Sciences \\ Nanyang Technological University \\ 21 Nanyang Link, Singapore 637371
}

January 12, 2021

\begin{abstract}
We derive sufficient conditions for the differentiability of all orders for the flow of stochastic differential equations with jumps, and prove related $L^{p_{-}}$ integrability results for all orders. Our results extend similar results obtained in [Kun04] for first order differentiability and rely on the Burkholder-Davis-Gundy (BDG) inequality for time-inhomogeneous Poisson random measures on $\mathbb{R}_{+} \times \mathbb{R}$, for which we provide a new proof.
\end{abstract}

Keywords: Stochastic differential equations with jumps, moment bounds, Poisson random measures, stochastic flows, Markov semigroups.

Mathematics Subject Classification (2010): 60H10, 60H05, 60G44, 60J60, 60J75.

\section{Introduction}

In this paper we consider the regularity and integrability of all orders of the flow of Stochastic Differential Equations (SDEs) with jumps of the form

$$
d X_{t}(x)=r\left(t, X_{t}(x)\right) d t+\sigma\left(t, X_{t}(x)\right) d W_{t}+\int_{-\infty}^{\infty} g\left(t, X_{t^{-}}(x), y\right)\left(N(d t, d y)-\nu_{t}(d y) d t\right),
$$

with $X_{0}(x)=x$, where $X_{t^{-}}$stands for $\lim _{s}{ }_{t} X_{s}, g: \mathbb{R}_{+} \times \mathbb{R} \times \mathbb{R} \rightarrow \mathbb{R}$ is a deterministic measurable function and $\left(W_{t}\right)_{t \in \mathbb{R}_{+}}, N(d t, d y)$ are a standard Brownian motion

\footnotetext{
*jean-christophe.breton@univ-rennes1.fr

${ }^{\dagger}$ nprivault@ntu.edu.sg
} 
and a Poisson random measure on $\mathbb{R}^{+} \times \mathbb{R}$ with compensator $\nu_{t}(d y) d t$, generating a filtration $\left(\mathcal{F}_{t}\right)_{t \geq 0}$.

In the diffusion case, the smoothness of the solution flow $x \mapsto X_{t}(x)$ of stochastic differential equations of the form

$$
d X_{t}(x)=r\left(t, X_{t}(x)\right) d t+\sigma\left(t, X_{t}(x)\right) d W_{t}
$$

with $X_{0}(x)=x$, where $r: \mathbb{R}_{+} \times \mathbb{R} \rightarrow \mathbb{R}, \sigma: \mathbb{R}_{+} \times \mathbb{R} \rightarrow \mathbb{R}$ are deterministic coefficients, has been studied in [Kun84], [Kun90]. In Theorem II.4.4 of [Kun84] and Theorem 4.6.5 of [Kun90] it is shown that $x \mapsto X_{t}(x)$ is $k$ times continuously differentiable when the SDE coefficients of (1.2) are $\mathcal{C}^{k}$ functions with globally Lipschitz derivatives. Such results have been proved in the jump-diffusion case in [Kun04] in the case of first order differentiability, however, the extension to higher orders of differentiability is not trivial and requires us to use the framework of [BGJ87].

Our proofs rely on the Burkholder-Davis-Gundy (BDG) inequality, which states that for any martingale $\left(M_{t}\right)_{t \in \mathbb{R}_{+}}$and for all $p \geq 1$ we have

$$
\mathbb{E}\left[\left|M_{t}^{*}\right|^{p}\right] \leq C_{p} \mathbb{E}\left[[M, M]_{t}^{p / 2}\right], \quad t \in \mathbb{R}_{+}
$$

where $M_{t}^{*}=\sup _{s \in[0, t]}\left|M_{s}\right|$, with

$$
C_{p}=(10 p)^{p / 2} \text { for } 1 \leq p<2, C_{2}=2^{p}, \text { and } C_{p}=p^{p}\left(\frac{e}{2}\right)^{p / 2} \text { for } p>2
$$

cf. e.g. Theorem 4.2.12 of [Bic02] or Theorem 48 in Chapter IV of [Pro04]. When $p=2$ we have

$$
\mathbb{E}\left[\sup _{s \in[0, t]}\left|M_{s}\right|^{p}\right] \leq C_{p} \mathbb{E}\left[\langle M, M\rangle_{t}^{p / 2}\right], \quad t \in \mathbb{R}_{+},
$$

which implies the bound

$$
\mathbb{E}\left[\sup _{s \in[0, t]}\left|M_{s}\right|^{p}\right] \leq C_{p}\left(\mathbb{E}\left[\langle M, M\rangle_{t}\right]\right)^{p / 2}, \quad t \in \mathbb{R}_{+},
$$

for $p \in[1,2]$. However, (1.5) does not extend to any $p>2$, see e.g. Remark 357 page 384 of [Sit05]. For this reason we use Kunita's BDG inequality for jump processes, see Theorem 2.11 in [Kun04], which is recovered under a form similar to Corollary 2.14 
in [Hau11], see Lemma 2.1 and Corollary 2.2 below. This also extends related results obtained in the case of a standard Poisson process, in [HK05] see Corollary 1 and Lemma 1 therein, with application to the numerical solution of SDEs.

We proceed by deriving moment bounds for the solutions of SDEs with jumps of the form (1.1) in Theorem 3.1. Similar bounds have been obtained in Theorem 3.2 of [Kun04], however, here we work with random $\left(\mathcal{F}_{t}\right)_{t \geq 0}$-adapted coefficients and under weaker integrability conditions. Other moment bounds for SDEs with jumps have been derived using (1.3) in various works, see for example Lemma 1 in [ZWL15] or Lemma 2.2 in [ZZ16]. However, those approaches rely on the incorrect assumption that (1.5) holds for any $p \geq 1$. Nevertheless, (1.5) is valid for $p=2$, and in this case it has been used in [ZZ16] to derive bounds on $\mathbb{E}\left[\sup _{s \in[0, t]}\left|M_{s}\right|^{p}\right]$ for $p>1$, see the proof of Theorem 2.1 therein and also [PW19] for an application of Kunita's BDG inequality to SIR population models for any $p>2$.

The proofs of Proposition 4.1 on the existence of the flow derivatives and of Theorem 5.1 on their $L^{p}$ integrability rely on Theorems 6-29 and 6-44 of [BGJ87]. For this reason, in Sections 4 and 5 we will assume that the compensators $\nu_{t}(d y), t \in \mathbb{R}_{+}$, in (1.1) are dominated by a (deterministic) measure $\eta$ on $\mathbb{R}$, i.e.

$$
\nu_{t}(A) \leq \eta(A), \quad A \in \mathcal{B}(\mathbb{R})
$$

where $\mathcal{B}(\mathbb{R})$ denotes the Borel $\sigma$-algebra of $\mathbb{R}$, in addition to the following Assumption $\left(A_{n}\right)$, see $\left(A^{\prime}-r\right)$ page 60 in [BGJ87].

Assumption $\left(A_{n}\right):$ For every $t \in \mathbb{R}_{+}$, the functions $r(t, \cdot): \mathbb{R} \rightarrow \mathbb{R}, \sigma(t, \cdot): \mathbb{R} \rightarrow \mathbb{R}$ and $g(t, \cdot): \mathbb{R} \times \mathbb{R} \rightarrow \mathbb{R}$ are $\mathcal{C}^{n}$-differentiable and there is a constant $C>0$ such that

$$
\left|\frac{\partial^{k} r}{\partial x^{k}}(t, x)\right| \leq C, \quad\left|\frac{\partial^{k} \sigma}{\partial x^{k}}(t, x)\right| \leq C, \quad\left|\frac{\partial^{k+l} g}{\partial x^{k} \partial y^{l}}(t, x, y)\right| \leq C,
$$

for all $k, l=1, \ldots, n$ with $1 \leq k+l \leq n$, and a function $\theta \in \bigcap_{q \geq 2} L^{q}(\mathbb{R}, \eta)$ such that

$$
\left|\frac{\partial^{k} g}{\partial x^{k}}(t, x, y)\right| \leq C \theta(y), \quad x, y \in \mathbb{R}, \quad k=1, \ldots, n .
$$


Although the results of [BGJ87] are stated for time-homogeneous SDE coefficients in (1.1), they remain valid under our time-inhomogeneous Assumption $\left(A_{n}\right)$. For this, we note that Theorems 6-20, 6-24, 6-29 and 6-44 in [BGJ87] all rely on Lemma 5.1 page 44 therein, which extends to the time-inhomogeneous case thanks to the domination condition (1.6), see Lemma A.14 of [BJ83] and Theorem 2.1 in [Bic81].

Under (1.6) and Assumption $\left(A_{n}\right)$, in Theorem 5.1 we provide sufficient conditions for the flow derivative

$$
X_{t}^{(n)}(x):=\frac{\partial^{n} X_{t}}{\partial x^{n}}(x)
$$

to exist and belong to $L^{p}(\Omega)$ uniformly in $(x, t) \in \mathbb{R} \times[0, T]$, i.e.,

$$
\sup _{x \in \mathbb{R}} \mathbb{E}\left[\sup _{t \in[0, T]}\left|X_{t}^{(n)}(x)\right|^{p}\right]<\infty
$$

for given orders of derivation $n \geq 1$ and of integrability $p \geq 1$.

Flow regularity results up to order four of differentiability have also been obtained in [PT97] based on a different version of the BDG inequality for Lévy processes with stochastic integrands depending only on time (see Lemma 4.1 page 409 of [PT97]), with application to the convergence of the Euler method.

As a consequence of (1.8), when $f(x):=(x-K)^{+}$is the (Lipschitz) payoff function of a European call option, we can also express the Delta, or first derivative of the option price with respect to the underlying price $x$ as

$$
\frac{\partial P_{t} f}{\partial x}(x)=\mathbb{E}\left[\mathbf{1}_{\left\{X_{t}(x) \geq K\right\}} X_{t}^{(1)}(x)\right], \quad t \in \mathbb{R}_{+}, \quad x \in \mathbb{R} .
$$

More generally, given the transition semigroup $\left(P_{t}\right)_{t \geq 0}$ of $\left(X_{t}(x)\right)_{t \geq 0}$, defined as

$$
P_{t} f(x):=\mathbb{E}\left[f\left(X_{t}(x)\right)\right], \quad t \in \mathbb{R}_{+}, \quad x \in \mathbb{R},
$$

we deduce that for any $f \in \mathcal{C}_{b}^{\infty}(\mathbb{R})$ the function $x \mapsto P_{t} f(x)$ is $\mathcal{C}^{\infty}$, with

$$
\frac{\partial^{n} P_{t} f}{\partial x^{n}}(x)=\sum_{\pi \in \Pi[n]} \mathbb{E}\left[\frac{\partial^{|\pi|} f}{\partial x^{|\pi|}}\left(x, X_{t}(x)\right) \prod_{B \in \pi} X_{t}^{(|B|)}(x)\right], \quad t \in \mathbb{R}_{+}, \quad x \in \mathbb{R},
$$


by the Faà di Bruno formula, where the sum over $\pi$ runs in the set $\Pi[n]$ of all partitions of $\{1,2, \ldots, n\}$, the product over $B \in \pi$ runs in all blocks $B$ in the partition $\pi$, and $|A|$ stands for the cardinality of the set $A$. The moment bounds obtained in this paper are also applied to the derivation of distance estimates between jump-diffusion processes in [BP20].

We proceed as follows. In Section 2 we derive two versions of the BDG inequality with jumps, similarly to Theorem 2.11 [Kun04] and to Corollary 2.14 of [Hau11], and we show that they can be unified in Corollary 2.2. This is followed by moment bounds

for SDEs in Section 3. In Section 4 we deal with the flow derivatives $X_{t}^{(n)}(x)$ by noting that they satisfy an affine SDE, for which moment bounds can be obtained from Theorem 3.1, see Proposition 4.1. Next, in Section 5 we present our result on moment bounds for flow derivatives, see Theorem 5.1.

\section{Burkholder-Davis-Gundy inequality with jumps}

Our moment bounds rely on a version of the BDG inequality (1.3) which uses the compensator $\langle M, M\rangle$ of $M$ instead of its bracket $[M, M]$. Consider the compensated Poisson stochastic integral process

$$
K_{t}:=\int_{0}^{t} \int_{-\infty}^{\infty} g_{s}(y)\left(N(d s, d y)-\nu_{s}(d y) d s\right), \quad t \in \mathbb{R}_{+}
$$

of the predictable integrand $\left(g_{s}(y)\right)_{(s, y) \in \mathbb{R}_{+} \times \mathbb{R}}$, where $N$ is a Poisson random measure on $\mathbb{R}_{+} \times \mathbb{R}$ with compensator $\nu_{t}(d y) d t$. When $p=2$, the BDG inequality (1.3) shows that

$$
\mathbb{E}\left[\left(K_{t}^{*}\right)^{2}\right] \leq 2 \mathbb{E}\left[\int_{0}^{t} \int_{-\infty}^{\infty}\left(g_{s}(y)\right)^{2} N(d s, d y)\right]=2 \mathbb{E}\left[\int_{0}^{t} \int_{-\infty}^{\infty}\left(g_{s}(y)\right)^{2} \nu_{s}(d y) d s\right],
$$

where $K_{t}^{*}=\sup _{s \in[0, t]}\left|K_{s}\right|$, since $t \mapsto \int_{0}^{t} \int_{-\infty}^{\infty}\left(g_{s}(y)\right)^{2}\left(N(d s, d y)-\nu_{s}(d y) d s\right)$ is a martingale. In particular, for any $p \in[1,2]$ we have

$$
\begin{aligned}
\mathbb{E}\left[\left(K_{t}^{*}\right)^{p}\right] & \leq\left(\mathbb{E}\left[\left(K_{t}^{*}\right)^{2}\right]\right)^{p / 2} \\
& \leq 2^{p / 2}\left(\mathbb{E}\left[\int_{0}^{t} \int_{-\infty}^{\infty}\left(g_{s}(y)\right)^{2} \nu_{s}(d y) d s\right]\right)^{p / 2}, \quad t \in \mathbb{R}_{+} .
\end{aligned}
$$


Lemma 2.1 below extends the BDG inequality to $p>2$ with explicit bounding constants, in relation to the BDG inequality stated for $1<p \leq 2$ in Corollary 2.14 of [Hau11].

Lemma 2.1 Consider the compensated Poisson stochastic integral process $\left(K_{t}\right)_{t \in \mathbb{R}_{+}}$in

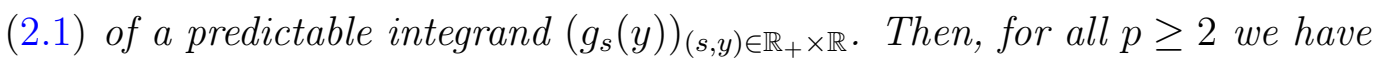

$$
\begin{aligned}
\mathbb{E}\left[\left(K_{t}^{*}\right)^{p}\right] \leq & \frac{2}{p}(40 p)^{p / 2}\left(\frac{p^{2} e}{2}\right)^{p\left(\log _{2} p\right) / 2} \mathbb{E}\left[\int_{0}^{t} \int_{-\infty}^{\infty}\left|g_{s}(y)\right|^{p} \nu_{s}(d y) d s\right] \\
& +2^{p} \sum_{k=1}^{\left\lceil\log _{2} p\right\rceil-1} \frac{p^{p k}}{2^{k}}\left(\frac{e}{2}\right)^{k p / 2} \mathbb{E}\left[\left(\int_{0}^{t} \int_{-\infty}^{\infty}\left(g_{s}(y)\right)^{2^{k}} \nu_{s}(d y) d s\right)^{p / 2^{k}}\right], \quad t \in \mathbb{R}_{+} .
\end{aligned}
$$

Proof. For $r \geq 1$ let

$$
K_{t}^{(r)}:=\int_{0}^{t} \int_{-\infty}^{\infty}\left(g_{s}(y)\right)^{r}\left(N(d s, d y)-\nu_{s}(d y) d s\right),
$$

with $K_{t}^{(1)}=K_{t}, t \in \mathbb{R}_{+}$. When $p>2$, since $x \mapsto|x|^{p / 2}$ is convex, (1.3) entails

$$
\begin{aligned}
& \mathbb{E}\left[\left(\left(K_{t}^{(r)}\right)^{*}\right)^{p}\right] \leq p^{p}\left(\frac{e}{2}\right)^{p / 2} \mathbb{E}\left[\left(\int_{0}^{t} \int_{-\infty}^{\infty}\left(g_{s}(y)\right)^{2 r} N(d s, d y)\right)^{p / 2}\right] \\
& =p^{p}\left(\frac{e}{2}\right)^{p / 2} \mathbb{E}\left[\left|\int_{0}^{t} \int_{-\infty}^{\infty}\left(g_{s}(y)\right)^{2 r}\left(N(d s, d y)-\nu_{s}(d y)\right) d s+\int_{0}^{t} \int_{-\infty}^{\infty}\left(g_{s}(y)\right)^{2 r} \nu_{s}(d y) d s\right|^{p / 2}\right] \\
& \leq 2^{p / 2-1} p^{p}\left(\frac{e}{2}\right)^{p / 2} \mathbb{E}\left[\left|\int_{0}^{t} \int_{-\infty}^{\infty}\left(g_{s}(y)\right)^{2 r}\left(N(d s, d y)-\nu_{s}(d y) d s\right)\right|^{p / 2}\right] \\
& +2^{p / 2-1}\left(\frac{e}{2}\right)^{p / 2} \mathbb{E}\left[\left|\int_{0}^{t} \int_{-\infty}^{\infty}\left(g_{s}(y)\right)^{2 r} \nu_{s}(d y) d s\right|^{p / 2}\right] \\
& \leq 2^{p / 2-1} p^{p}\left(\frac{e}{2}\right)^{p / 2} \mathbb{E}\left[\left(\left(K_{t}^{(2 r)}\right)^{*}\right)^{p / 2}\right]+2^{p / 2-1} p^{p}\left(\frac{e}{2}\right)^{p / 2} \mathbb{E}\left[\left(\int_{0}^{t} \int_{-\infty}^{\infty}\left(g_{s}(y)\right)^{2 r} \nu_{s}(d y) d s\right)^{p / 2}\right] .
\end{aligned}
$$

The recursive application of the bound (2.4) starting from $K_{t}^{(1)}=K_{t}$ yields

$$
\begin{aligned}
\mathbb{E}\left[\left(K_{t}^{*}\right)^{p}\right] \leq & p^{n p}\left(\frac{e}{2}\right)^{p n / 2}\left(\prod_{j=1}^{n} 2^{p / 2^{j}-1}\right) \mathbb{E}\left[\left(\left(K_{t}^{\left(2^{n}\right)}\right)^{*}\right)^{p / 2^{n}}\right] \\
& +\sum_{k=1}^{n} p^{k p}\left(\frac{e}{2}\right)^{p n / 2}\left(\prod_{j=1}^{k} 2^{p / 2^{j}-1}\right) \mathbb{E}\left[\left(\int_{0}^{t} \int_{-\infty}^{\infty}\left(g_{s}(y)\right)^{2^{k}} \nu_{s}(d y) d s\right)^{p / 2^{k}}\right] .
\end{aligned}
$$


Taking $n=\left\lceil\log _{2} p\right\rceil-1$, i.e. $p \in\left(2^{n}, 2^{n+1}\right]$, by $(1.3)$ we have

$$
\begin{aligned}
\mathbb{E}\left[\left(\left(K_{t}^{\left(2^{n}\right)}\right)^{*}\right)^{p / 2^{n}}\right] & \leq(10 p)^{p / 2} \mathbb{E}\left[\left(\int_{0}^{t} \int_{-\infty}^{\infty}\left(g_{s}(y)\right)^{2^{n+1}} N(d s, d y)\right)^{p / 2^{n+1}}\right] \\
& \leq(10 p)^{p / 2} \mathbb{E}\left[\int_{0}^{t} \int_{-\infty}^{\infty}\left|g_{s}(y)\right|^{p} N(d s, d y)\right] \\
& =(10 p)^{p / 2} \mathbb{E}\left[\int_{0}^{t} \int_{-\infty}^{\infty}\left|g_{s}(y)\right|^{p} \nu_{s}(d y) d s\right],
\end{aligned}
$$

since $t \mapsto \int_{0}^{t} \int_{-\infty}^{\infty}\left(g_{s}(y)\right)^{2}\left(N(d s, d y)-\nu_{s}(d y) d s\right)$ is a martingale, where we used the fact that

$$
\|a\|_{\ell^{2}}:=\left(\sum_{k=0}^{\infty}\left(a_{k}\right)^{2}\right)^{1 / 2} \leq\left(\sum_{k=0}^{\infty}\left(a_{k}\right)^{q}\right)^{1 / q}=:\|a\|_{\ell^{q}}
$$

for any real sequence $\left(a_{k}\right)_{k \in \mathbb{N}}$ and $1 \leq q=p / 2^{n} \leq 2$ as on page 410 after Equation (22) in [PT97], which allows us to conclude to (2.3).

From Lemma 2.1 we recover the following version of the Kunita's BDG inequality with jumps, cf. Theorem 2.11 of [Kun04] and Theorem 4.4.23 of [App09].

Corollary 2.2 Consider the compensated Poisson stochastic integral process

$$
K_{t}:=x+\int_{0}^{t} u_{s} d s+\int_{0}^{t} v_{s} d W_{s}+\int_{0}^{t} \int_{-\infty}^{\infty} g_{s}(y)\left(N(d s, d y)-\nu_{s}(d y) d s\right), \quad t \in \mathbb{R}_{+},
$$

of the predictable integrands $\left(u_{s}\right)_{s \in \mathbb{R}_{+}},\left(v_{s}\right)_{s \in \mathbb{R}_{+}},\left(g_{s}(y)\right)_{(s, y) \in \mathbb{R}_{+} \times \mathbb{R}}$. Then, for all $p \geq 2$ and $T \in \mathbb{R}_{+}$we have

$$
\begin{aligned}
& \mathbb{E}\left[\left(K_{T}^{*}\right)^{p}\right] \\
& \leq 2^{2 p-2}\left(|x|^{p}+\mathbb{E}\left[\left(\int_{0}^{T}\left|u_{t}\right| d t\right)^{p}\right]+C_{p} \mathbb{E}\left[\left(\int_{0}^{T}\left|v_{t}\right|^{2} d t\right)^{p / 2}\right]\right. \\
& \left.\quad+\widetilde{C}_{p} \mathbb{E}\left[\int_{0}^{T} \int_{-\infty}^{\infty}\left|g_{t}(y)\right|^{p} \nu_{t}(d y) d t\right]+\widetilde{C}_{p} \mathbb{E}\left[\left(\int_{0}^{T} \int_{-\infty}^{\infty}\left|g_{t}(y)\right|^{2} \nu_{t}(d y) d t\right)^{p / 2}\right]\right),
\end{aligned}
$$

where $C_{p}$ is defined in (1.4), and

$$
\widetilde{C}_{p}=\frac{2}{p}(40 p)^{p / 2}\left(\frac{p^{2} e}{2}\right)^{p\left(\log _{2} p\right) / 2}+2^{p} \sum_{k=1}^{\left\lceil\log _{2} p\right\rceil-1} \frac{p^{p k}}{2^{k}}\left(\frac{e}{2}\right)^{k p / 2} \leq 2^{p} p^{p \log _{2} p}\left(2+\left(10 e^{\left\lceil\log _{2} p\right\rceil}\right)^{p / 2}\right) .
$$

Proof. By the convexity of $\mathbb{R} \ni x \mapsto|x|^{p}$, we have

$$
\mathbb{E}\left[\sup _{s \in[0, T]}\left|K_{t^{-}}\right|^{p}\right] \leq 4^{p-1}\left(x^{p}+\mathbb{E}\left[\sup _{t \in[0, T]}\left|\int_{0}^{t} u_{s} d s\right|^{p}\right]+\mathbb{E}\left[\sup _{t \in[0, T]}\left|\int_{0}^{t} v_{s} d W_{s}\right|^{p}\right]\right.
$$




$$
\left.+\mathbb{E}\left[\sup _{t \in[0, T]}\left|\int_{0}^{t^{-}} \int_{-\infty}^{\infty} g_{s^{-}}(z)\left(N(d s, d z)-\nu_{s}(d z) d s\right)\right|^{p}\right]\right) .
$$

Further, by the (standard) BDG inequality for Brownian stochastic integrals, the Jensen inequality for the uniform measure on $[0, t]$ and the Fubini theorem, we find:

$$
\mathbb{E}\left[\sup _{t \in[0, T]}\left|\int_{0}^{t} v_{s} d W_{s}\right|^{p}\right] \leq C_{p} \mathbb{E}\left[\left(\int_{0}^{T}\left|v_{s}\right|^{2} d s\right)^{p / 2}\right] .
$$

Regarding the jump term, by the log-convexity in $p$ of the $L^{p}$ norms, taking $n=$ $\left\lceil\log _{2} p\right\rceil-1$, i.e. $p \in\left(2^{n}, 2^{n+1}\right]$, and $\theta \in(0,1)$ such that $2^{-k}=(1-\theta) / p+\theta / 2$, we have

$$
\begin{aligned}
& \mathbb{E} {\left[\left(\int_{0}^{T} \int_{-\infty}^{\infty}\left(g_{t}(y)\right)^{2^{k}} \nu_{t}(d y) d t\right)^{p / 2^{k}}\right] } \\
& \leq \mathbb{E}\left[\left(\int_{0}^{T} \int_{-\infty}^{\infty}\left(g_{t}(y)\right)^{p} \nu_{t}(d y) d t\right)^{1-\theta}\left(\int_{0}^{T} \int_{-\infty}^{\infty}\left(g_{t}(y)\right)^{2} \nu_{t}(d y) d t\right)^{\theta p / 2}\right] \\
& \leq\left(\mathbb{E}\left[\int_{0}^{T} \int_{-\infty}^{\infty}\left(g_{t}(y)\right)^{p} \nu_{t}(d y) d t\right]\right)^{1-\theta}\left(\mathbb{E}\left[\left(\int_{0}^{T} \int_{-\infty}^{\infty}\left(g_{t}(y)\right)^{2} \nu_{t}(d y) d t\right)^{p / 2}\right]\right)^{\theta} \\
& \leq(1-\theta) \mathbb{E}\left[\int_{0}^{T} \int_{-\infty}^{\infty}\left(g_{t}(y)\right)^{p} \nu_{t}(d y) d t\right]+\theta \mathbb{E}\left[\left(\int_{0}^{T} \int_{-\infty}^{\infty}\left(g_{t}(y)\right)^{2} \nu_{t}(d y) d t\right)^{p / 2}\right] \\
& \leq \mathbb{E}\left[\int_{0}^{T} \int_{-\infty}^{\infty}\left(g_{t}(y)\right)^{p} \nu_{t}(d y) d t\right]+\mathbb{E}\left[\left(\int_{0}^{T} \int_{-\infty}^{\infty}\left(g_{t}(y)\right)^{2} \nu_{t}(d y) d t\right)^{p / 2}\right], \quad T \mathbb{R}_{+},
\end{aligned}
$$

$k=1, \ldots, n$, after using the Hölder inequality and the bound $x^{1-\theta} y^{\theta} \leq(1-\theta) x+\theta y$, $x, y \geq 0$. Hence, substituting this bound in (2.3), we obtain

$$
\begin{aligned}
\mathbb{E}\left[\sup _{t \in[0, T]}\left|\int_{0}^{t^{-}} \int_{-\infty}^{\infty} g_{s^{-}}(z)\left(N(d s, d z)-\nu_{s}(d z) d s\right)\right|^{p}\right] \\
\leq \frac{2^{p+1}}{p} p^{p \log _{2} p}(10 p)^{p / 2}\left(\frac{e}{2}\right)^{p\left(\log _{2} p\right) / 2} \mathbb{E}\left[\int_{0}^{T} \int_{-\infty}^{\infty}\left(g_{t}(y)\right)^{p} \nu_{t}(d y) d t\right] \\
\quad+2^{p} \sum_{k=1}^{\left\lceil\log _{2} p\right\rceil-1} \frac{p^{p k}}{2^{k}}\left(\frac{e}{2}\right)^{k p / 2} \mathbb{E}\left[\left(\int_{0}^{T} \int_{-\infty}^{\infty}\left(g_{t}(y)\right)^{2^{k}} \nu_{t}(d y) d t\right)^{p / 2^{k}}\right] \\
\leq \widetilde{C}_{p}\left(\mathbb{E}\left[\int_{0}^{T} \int_{-\infty}^{\infty}\left(g_{t}(y)\right)^{p} \nu_{t}(d y) d t\right]+\mathbb{E}\left[\left(\int_{0}^{T} \int_{-\infty}^{\infty}\left(g_{t}(y)\right)^{2} \nu_{t}(d y) d t\right)^{p / 2}\right]\right) .
\end{aligned}
$$


The following consequence of Corollary 2.2 recovers Corollary 2.12 in [Kun04] using the Hölder inequality.

Corollary 2.3 Consider the compensated Poisson stochastic integral process $\left(K_{t}\right)_{t \in[0, T]}$ in (2.5). For all $p \geq 2$ and $T \in \mathbb{R}_{+}$we have

$$
\begin{aligned}
& \mathbb{E}\left[\left(K_{T}^{*}\right)^{p}\right] \leq 2^{2 p-2}\left(|x|^{p}+T^{p-1} \mathbb{E}\left[\int_{0}^{T}\left|u_{t}\right|^{p} d t\right]+C_{p} T^{p / 2-1} \mathbb{E}\left[\int_{0}^{T}\left|v_{t}\right|^{p} d t\right]\right. \\
& \left.+\widetilde{C}_{p} \mathbb{E}\left[\int_{0}^{T} \int_{-\infty}^{\infty}\left|g_{t}(y)\right|^{p} \nu_{t}(d y) d t\right]+T^{p / 2-1} \widetilde{C}_{p} \mathbb{E}\left[\int_{0}^{T}\left(\int_{-\infty}^{\infty}\left|g_{t}(y)\right|^{2} \nu_{t}(d y)\right)^{p / 2} d t\right]\right) .
\end{aligned}
$$

When the integrand $g_{t}(y)$ satisfies $\left|g_{t}(y)\right| \leq|f(y)|\left|g_{t}\right|$ where $f(y)$ is a deterministic function of $y \in \mathbb{R},\left(g_{t}\right)_{t \in \mathbb{R}_{+}}$is an $\left(\mathcal{F}_{t}\right)_{t \geq 0}$-adapted process, and $\left(\nu_{t}(d y)\right)_{t \in \mathbb{R}_{+}}=\nu(d y)$, $t \in \mathbb{R}_{+}$, is the intensity measure of a time-homogeneous Poisson point process, Corollary 2.3 yields

$$
\begin{aligned}
& \mathbb{E}\left[\left(K_{T}^{*}\right)^{p}\right] \leq 2^{2 p-2}\left(|x|^{p}+T^{p-1} \mathbb{E}\left[\int_{0}^{T}\left|u_{t}\right|^{p} d t\right]+C_{p} T^{p / 2-1} \mathbb{E}\left[\int_{0}^{T}\left|v_{t}\right|^{p} d t\right]\right. \\
& \left.+\widetilde{C}_{p}\left(\int_{-\infty}^{\infty}|f(y)|^{p} \nu(d y)+T^{p / 2-1}\left(\int_{-\infty}^{\infty}|f(y)|^{2} \nu(d y)\right)^{p / 2}\right) \mathbb{E}\left[\int_{0}^{T}\left|g_{t}\right|^{p} d t\right]\right),
\end{aligned}
$$

which recovers related versions of the BDG inequality with jumps such as Lemma 5.2 of [BC86] which is stated for $p=2^{n}, n \geq 1$, or Lemma 4.1 of [PT97] which is stated for $p \geq 2$ using a related recursion. We also refer the reader to Lemma A.14 of [BJ83] and to the proof of Theorem 2.1 in [Bic81], or to [LLP80] and [Pra83], for other versions of the BDG inequality with jumps.

\section{Moment bounds for SDE solutions}

In this section, we derive moment bounds for jump-diffusion SDEs, based on the BDG inequality with jumps given in Corollary 2.2 .

The following result provides moment bounds in $L^{p}(\Omega), p \geq 2$, on the solution of SDEs of the form

$$
d X_{t}=a_{t}\left(X_{t}\right) d t+b_{t}\left(X_{t}\right) d W_{t}+\int_{-\infty}^{\infty} c_{t^{-}}\left(z, X_{t^{-}}\right)\left(N(d t, d z)-\nu_{t}(d z) d t\right),
$$


whose existence and uniqueness of solutions follows by standard arguments, see e.g. Theorem 3.1 in [Kun04]. In contrast with Theorem 3.2 of [Kun04], we work with random $\left(\mathcal{F}_{t}\right)_{t \geq 0}$-adapted coefficients and under weaker integrability conditions as Condition (3.2) in [Kun04] requires integrability of all orders. We let $\|X\|_{\infty}$ stand for the $L^{\infty}(\Omega)$ norm of a random variable $X$.

Theorem 3.1 Let $p \geq 2$ and consider the solution $\left(X_{t}\right)_{t \geq 0}$ of the one-dimensional solution of the jump-diffusion SDE (3.1), where the coefficients $\left(a_{t}(x)\right)_{t \in[0, T]},\left(b_{t}(x)\right)_{t \in[0, T]}$, $\left(c_{t}(z, x)\right)_{t \in[0, T]}$ are $\left(\mathcal{F}_{t}\right)_{t \geq 0}$-adapted processes such that

$$
\left|a_{t}(x)-a_{t}(y)\right| \leq a_{t}|x-y|, \quad\left|b_{t}(x)-b_{t}(y)\right| \leq b_{t}|x-y|, \quad x, y \in \mathbb{R}
$$

where $\left(a_{t}(0)\right)_{t \in[0, T]},\left(b_{t}(0)\right)_{t \in[0, T]} \in L^{p}(\Omega \times[0, T]),\left(a_{t}\right)_{t \in[0, T]},\left(b_{t}\right)_{t \in[0, T]} \in L^{p}\left([0, T], L^{\infty}(\Omega)\right)$, and

$$
\left|c_{t}(z, x)-c_{t}(z, y)\right| \leq c_{t}(z)|x-y|, \quad x, y \in \mathbb{R}
$$

where

$$
\int_{0}^{T}\left\|\int_{-\infty}^{\infty}\left|c_{t}(z)\right|^{2} \nu_{t}(d z)\right\|_{\infty}^{p / 2} d t<\infty, \quad \int_{0}^{T}\left\|\int_{-\infty}^{\infty}\left|c_{t}(z)\right|^{p} \nu_{t}(d z)\right\|_{\infty} d t<\infty
$$

and

$$
\mathbb{E}\left[\int_{0}^{T}\left(\int_{-\infty}^{\infty}\left|c_{t}(z, 0)\right|^{2} \nu_{t}(d z)\right)^{p / 2} d t\right]<\infty, \mathbb{E}\left[\int_{0}^{T} \int_{-\infty}^{\infty}\left|c_{t}(z, 0)\right|^{p} \nu_{t}(d z) d t\right]<\infty
$$

Then we have

$$
\mathbb{E}\left[\sup _{t \in[0, T]}\left|X_{t}\right|^{p}\right]<C(p, T)<\infty
$$

where $C(p, T)$ depends on the above norms of $a, b, c, u, v, w$.

Proof. We have

$$
\mathbb{E}\left[\int_{0}^{T}\left|a_{t}\left(X_{t}\right)\right|^{p} d t\right] \leq 2^{p-1}\left(\int_{0}^{T}\left\|a_{t}\right\|_{\infty}^{p} \mathbb{E}\left[\left|X_{t}\right|^{p}\right] d t+\mathbb{E}\left[\int_{0}^{T}\left|a_{t}(0)\right|^{p} d t\right]\right),
$$

and

$$
\mathbb{E}\left[\int_{0}^{T}\left|b_{t}\left(X_{t}\right)\right|^{p} d t\right] \leq 2^{p-1}\left(\int_{0}^{T}\left\|b_{t}\right\|_{\infty}^{p} \mathbb{E}\left[\left|X_{t}\right|^{p}\right] d t+\mathbb{E}\left[\int_{0}^{T}\left|b_{t}(0)\right|^{p} d t\right]\right) .
$$


Regarding the jump term, we note that

$$
\begin{aligned}
& \widetilde{C}_{p} T^{p / 2-1} \mathbb{E}\left[\int_{0}^{T}\left(\int_{-\infty}^{\infty}\left|c_{t}\left(z, X_{t}\right)\right|^{2} \nu_{t}(d z)\right)^{p / 2} d t\right]+\widetilde{C}_{p} \mathbb{E}\left[\int_{0}^{T} \int_{-\infty}^{\infty}\left|c_{t}\left(z, X_{t}\right)\right|^{p} \nu_{t}(d z) d t\right] \\
& \leq 2^{p} \widetilde{C}_{p} T^{p / 2-1} \mathbb{E}\left[\int_{0}^{T}\left(\int_{-\infty}^{\infty}\left|c_{t}(z)\right|^{2}\left|X_{t}\right|^{2} \nu_{t}(d z)+\int_{-\infty}^{\infty}\left|c_{t}(z, 0)\right|^{2} \nu_{t}(d z)\right)^{p / 2} d t\right] \\
& \quad+2^{p-1} \widetilde{C}_{p} \mathbb{E}\left[\int_{0}^{T} \int_{-\infty}^{\infty}\left(\left|c_{t}(z)\right|^{p}\left|X_{t}\right|^{p}+\left|c_{t}(z, 0)\right|^{p}\right) \nu_{t}(d z) d t\right] \\
& \leq 2^{3 p / 2-1} \widetilde{C}_{p} T^{p / 2-1} \mathbb{E}\left[\int_{0}^{T}\left(\left(\int_{-\infty}^{\infty}\left|c_{t}(z)\right|^{2} \nu_{t}(d z)\right)^{p / 2}\left|X_{t}\right|^{p}+\left(\int_{-\infty}^{\infty}\left|c_{t}(z, 0)\right|^{2} \nu_{t}(d z)\right)^{p / 2}\right) d t\right] \\
& \quad+2^{p-1} \widetilde{C}_{p} \mathbb{E}\left[\int_{0}^{T} \int_{-\infty}^{\infty}\left|c_{t}(z)\right|^{p} \nu_{t}(d z)\left|X_{t}\right|^{p} d t\right]+2^{p-1} \widetilde{C}_{p} \mathbb{E}\left[\int_{0}^{T} \int_{-\infty}^{\infty}\left|c_{t}(z, 0)\right|^{p} \nu_{t}(d z) d t\right] \\
& \leq 2^{3 p / 2-1} \widetilde{C}_{p} T^{p / 2-1} \int_{0}^{T} \|\left.\int_{-\infty}^{\infty}\left|c_{t}(z)\right|^{2} \nu_{t}(d z)\right|_{\infty} ^{p / 2} \mathbb{E}\left[X_{t}^{p}\right] d t \\
& \quad+2^{3 p / 2-1} \widetilde{C}_{p} T^{p / 2-1} \mathbb{E}\left[\int_{0}^{T}\left(\int_{-\infty}^{\infty}\left|c_{t}(z, 0)\right|^{2} \nu_{t}(d z)\right)^{p / 2} d t\right] \\
& \quad+2^{p-1} \widetilde{C}_{p} \int_{0}^{T}\left\|\int_{-\infty}^{\infty}\left|c_{t}(z)\right|^{p} \nu_{t}(d z) \mid\right\|_{\infty} \mathbb{E}\left[X_{t}^{p}\right] d t+2^{p-1} \widetilde{C}_{p} \mathbb{E}\left[\int_{0}^{T} \int_{-\infty}^{\infty}\left|c_{t}(z, 0)\right|^{p} \nu_{t}(d z) d t\right] .
\end{aligned}
$$

Hence by the BDG inequality of Corollary 2.3 and the bounds (3.4), (3.5), (3.6), setting

$$
\begin{aligned}
& F(T):=4^{p-1}\left(x^{p}+(2 T)^{p-1} \mathbb{E}\left[\int_{0}^{T}\left|a_{t}(0)\right|^{p} d t\right]+2^{p-1} C_{p} T^{p / 2-1} \mathbb{E}\left[\int_{0}^{T}\left|b_{t}(0)\right|^{p} d t\right]\right. \\
& \left.+2^{p-1} \widetilde{C}_{p}\left(2^{p / 2} T^{p / 2-1} \mathbb{E}\left[\int_{0}^{T}\left(\int_{-\infty}^{\infty}\left|c_{t}(z, 0)\right|^{2} \nu_{t}(d z)\right)^{p / 2} d t\right]+\mathbb{E}\left[\int_{0}^{T} \int_{-\infty}^{\infty}\left|c_{t}(z, 0)\right|^{p} \nu_{t}(d z) d t\right]\right)\right),
\end{aligned}
$$

and

$$
\begin{aligned}
& G(t):=4^{p-1}\left((2 T)^{p-1}\left\|a_{t}\right\|_{\infty}^{p}+2^{p-1} C_{p} T^{p / 2-1}\left\|b_{t}\right\|_{\infty}^{p}\right. \\
& \left.\quad+2^{3 p / 2-1} \widetilde{C}_{p} T^{p / 2-1}\left\|\int_{-\infty}^{\infty}\left|c_{t}(z)\right|^{2} \nu_{t}(d z)\right\|_{\infty}^{p / 2}+2^{p-1} \widetilde{C}_{p}\left\|\int_{-\infty}^{\infty}\left|c_{t}(z)\right|^{p} \nu_{t}(d z)\right\|_{\infty}\right),
\end{aligned}
$$

$t \in[0, T]$, we have

$$
\begin{aligned}
\mathbb{E}\left[\sup _{t \in[0, T]}\left|X_{t^{-}}\right|^{p}\right] & \leq F(T)+\mathbb{E}\left[\int_{0}^{T} G(t)\left|X_{t}\right|^{p} d t\right] \\
& \leq F(T)+\int_{0}^{T} G(t) \mathbb{E}\left[\sup _{s \in[0, t]}\left|X_{s}\right|^{p}\right] d t
\end{aligned}
$$


hence by the Grönwall lemma we find

$$
\mathbb{E}\left[\sup _{t \in[0, T]}\left|X_{t^{-}}\right|^{p}\right] \leq C(p, T):=F(T) \exp \left(\int_{0}^{T} G(t) d t\right),
$$

which is finite since $\left(a_{t}\right)_{t \in[0, T]},\left(b_{t}\right)_{t \in[0, T]} \in L^{p}\left([0, T], L^{\infty}(\Omega)\right)$ and (3.2)-(3.3) are in force. Since $X_{t^{-}}=X_{t}$ almost surely, the same bound follows for the moment of order $p$ of $X_{t}$.

Theorem 3.1 applies, in particular, to the solution $\left(X_{t}\right)_{t \geq 0}$ of the one-dimensional jump-diffusion affine SDE

$$
\begin{aligned}
d X_{t}= & u_{t} d t+a_{t} X_{t} d t+v_{t} d W_{t}+b_{t} X_{t} d W_{t} \\
& +\int_{-\infty}^{\infty} w_{t^{-}}(z)\left(N(d t, d z)-\nu_{t}(d z) d t\right)+X_{t^{-}} \int_{-\infty}^{\infty} c_{t^{-}}(z)\left(N(d t, d z)-\nu_{t}(d z) d t\right)
\end{aligned}
$$

by taking

$$
a_{t}(y)=a_{t} y+u_{t}, \quad b_{t}(y)=b_{t} y+v_{t}, \quad c_{t}(z, y)=c_{t}(z) y+w_{t}(z)
$$

where $u, v$ are in a certain $L^{p}$ space and $a, b, c$ are in $L^{\infty}$.

The following uniform version of Theorem 3.1 will be required in Section 4 . When the processes $a_{\alpha}, b_{\alpha}, c_{\alpha}$ all depend on a parameter $\alpha \in A$, the solution $\left(X_{\alpha, t}\right)_{t \geq 0}$ of the corresponding SDE (3.12) below enjoys the following uniform bound.

Corollary 3.2 Let $p \geq 2$. Assume that the coefficients $\left(a_{\alpha, t}(x)\right)_{t \in[0, T]},\left(b_{\alpha, t}(x)\right)_{t \in[0, T]}$, $\left(c_{\alpha, t}(z, x)\right)_{t \in[0, T]}$ are $\left(\mathcal{F}_{t}\right)_{t \geq 0}$-adapted processes such that

$$
\left|a_{\alpha, t}(x)-a_{\alpha, t}(y)\right| \leq a_{\alpha, t}|x-y|, \quad\left|b_{\alpha, t}(x)-b_{\alpha, t}(y)\right| \leq b_{\alpha, t}|x-y|, \quad x, y \in \mathbb{R},
$$

where $\left(a_{\alpha, t}(0)\right)_{t \in[0, T]},\left(b_{\alpha, t}(0)\right)_{t \in[0, T]} \in L^{p}(\Omega \times[0, T]),\left(a_{\alpha, t}\right)_{t \in[0, T]},\left(b_{\alpha, t}\right)_{t \in[0, T]} \in L^{p}\left([0, T], L^{\infty}(\Omega)\right)$, uniformly in $\alpha \in A$, with

$$
\left|c_{\alpha, t}(z, x)-c_{\alpha, t}(z, y)\right| \leq c_{\alpha, t}(z)|x-y|, \quad x, y \in \mathbb{R}
$$

with

$\sup _{\alpha \in A} \int_{0}^{T}\left\|\int_{-\infty}^{\infty}\left|c_{\alpha, t}(z)\right|^{2} \nu_{t}(d z)\right\|_{\infty}^{p / 2} d t<\infty, \sup _{\alpha \in A} \int_{0}^{T}\left\|\int_{-\infty}^{\infty}\left|c_{\alpha, t}(z)\right|^{p} \nu_{t}(d z)\right\|_{\infty} d t<\infty$, 
and

$\sup _{\alpha \in A} \mathbb{E}\left[\int_{0}^{T}\left(\int_{-\infty}^{\infty}\left|c_{\alpha, t}(z, 0)\right|^{2} \nu_{t}(d z)\right)^{p / 2} d t\right]<\infty, \sup _{\alpha \in A} \mathbb{E}\left[\int_{0}^{T} \int_{-\infty}^{\infty}\left|c_{\alpha, t}(z, 0)\right|^{p} \nu_{t}(d z) d t\right]<\infty$.

Then, for the solutions $X_{\alpha}$ of the $S D E$

$$
d X_{\alpha, t}=a_{\alpha, t}\left(X_{\alpha, t}\right) d t+b_{\alpha, t}\left(X_{\alpha, t}\right) d W_{t}+\int_{-\infty}^{\infty} c_{\alpha, t^{-}}\left(z, X_{\alpha, t^{-}}\right)\left(N(d t, d z)-\nu_{t}(d z) d t\right)
$$

$\alpha \in A$, we have

$$
\sup _{\alpha \in A} \mathbb{E}\left[\sup _{t \in[0, T]}\left|X_{\alpha, t}\right|^{p}\right]<C(p, T)<\infty
$$

where $C(p, T)$ depends on the above norms of $a_{\alpha}, b_{\alpha}, c_{\alpha}$, which are all assumed to be bounded uniformly in $\alpha \in A$.

Proof. Only the conclusion of the previous proof for Theorem 3.1 is required to be changed. The bound (3.7) still holds true for $X_{\alpha, t}$ with the functions

$$
\begin{aligned}
& F_{\alpha}(T):=4^{p-1}\left(x^{p}+(2 T)^{p-1} \mathbb{E}\left[\int_{0}^{T}\left|a_{\alpha, t}(0)\right|^{p} d t\right]+2^{p-1} C_{p} T^{p / 2-1} \mathbb{E}\left[\int_{0}^{T}\left|b_{\alpha, t}(0)\right|^{p} d t\right]\right. \\
& \left.+2^{p-1} \widetilde{C}_{p}\left(2^{p / 2} T^{p / 2-1} \mathbb{E}\left[\int_{0}^{T}\left(\int_{-\infty}^{\infty}\left|c_{\alpha, t}(z, 0)\right|^{2} \nu_{t}(d z)\right)^{p / 2} d t\right]+\mathbb{E}\left[\int_{-\infty}^{\infty}\left|c_{\alpha, t}(z, 0)\right|^{p} \nu_{t}(d z) d t\right]\right)\right)
\end{aligned}
$$

and

$$
\begin{aligned}
G_{\alpha}(t) & :=4^{p-1}\left((2 T)^{p-1}\left\|a_{\alpha, t}\right\|_{\infty}^{p}+2^{p-1} C_{p} T^{p / 2-1}\left\|b_{\alpha, t}\right\|_{\infty}^{p}\right. \\
& \left.\left.+2^{3 p / 2-1} \widetilde{C}_{p} T^{p / 2-1}\left\|\int_{-\infty}^{\infty}\left|c_{\alpha, t}(z)\right|^{2} \nu_{t}(d z)\right\|_{\infty}^{p / 2}+2^{p-1} \widetilde{C}_{p} \| \int_{-\infty}^{\infty}\left|c_{\alpha, t}(z)\right|^{p}\right) \nu_{t}(d z) \|_{\infty}\right),
\end{aligned}
$$

$t \in[0, T]$. Under the conditions of Corollary 3.2, we have

$$
\sup _{\alpha \in A} F_{\alpha}(T)<\infty, \quad \sup _{\alpha \in A} G_{\alpha}(t)<\infty, \quad t \in[0, T]
$$

and the conclusion (3.13) follows likewise.

\section{Flow derivatives}

In this section, we show that the derivatives of the flow $x \mapsto X_{t}(x)$ of the $\mathrm{SDE}(1.1)$ are solutions of an affine SDE. 
Convention. Given the gradient $\nabla_{z}=\left(\frac{\partial}{\partial z_{1}}, \ldots, \frac{\partial}{\partial z_{d}}\right)$ and $F: \mathbb{R}^{d} \rightarrow \mathbb{R}^{p}$, we denote $\nabla_{z} F(z)=\left(\frac{\partial F_{i}}{\partial z_{j}}(z)\right)_{\substack{1 \leq i \leq p \\ 1 \leq j \leq d}} \in M_{p, d}(\mathbb{R})$, and under the identification $M_{p, d}(\mathbb{R}) \sim \mathbb{R}^{p d}$, we write

$$
\nabla_{z}\left(\nabla_{z} F\right)(x)=\nabla_{z}^{2} F(x) \in M_{p d, d}(\mathbb{R}) \sim \mathbb{R}^{p d^{2}} .
$$

By successive differentiation of

$$
r\left(s, X_{s}(x)\right), \quad \sigma\left(s, X_{s}(x)\right), \quad g\left(s, X_{s}(x), y\right)
$$

with respect to $x$ and applying Theorem 6-29 of [BGJ87] recursively under Assumption $\left(A_{n}\right)$, we obtain the following result.

Proposition 4.1 Assume that (1.6) and $\left(A_{n}\right)$ hold for some $n \geq 1$. Then the flow $x \mapsto X_{t}(x)$ of the solution to the real SDE (1.1) is n times differentiable on $\mathbb{R}$ and, for $k=1, \ldots, n, X_{t}^{(k)}(x):=\frac{\partial^{k} X_{t}}{\partial x^{k}}(x)$ is solution of

$$
\begin{aligned}
& d X_{t}^{(k)}(x)=\left(\sum_{\pi \in \Pi[k]} \frac{\partial^{|\pi|} r}{\partial x^{|\pi|}}\left(t, X_{t}(x)\right) \prod_{B \in \pi} X_{t}^{(|B|)}(x)\right) d t \\
& \quad+\left(\sum_{\pi \in \Pi[k]} \frac{\partial^{|\pi|} \sigma}{\partial x^{|\pi|}}\left(t, X_{t}(x)\right) \prod_{B \in \pi} X_{t}^{(|B|)}(x)\right) d W_{t} \\
& \quad+\int_{-\infty}^{\infty}\left(\sum_{\pi \in \Pi[k]} \frac{\partial^{|\pi|} g}{\partial x^{|\pi|}}\left(t, X_{t}(x), y\right) \prod_{B \in \pi} X_{t}^{(|B|)}(x)\right)\left(N(d t, d y)-\nu_{t}(d y) d t\right),
\end{aligned}
$$

where, again, the sum over $\pi$ runs in the set $\Pi[k]$ of all partitions of $\{1,2, \ldots, k\}$, and the product over $B \in \pi$ runs in all blocks $B$ in the partition $\pi$.

Proof. For $n=0, X_{t}^{(0)}(x)=X_{t}(x)$ and (4.1) reduces to the $\operatorname{SDE}$ (1.1). For $n=1$, (4.1) is given by Theorem 6-29 in [BGJ87]:

$$
\begin{aligned}
d X_{t}^{(1)}(x)= & X_{t}^{(1)}(x) \frac{\partial r}{\partial x}\left(x, X_{t}(x)\right) d t+X_{t}^{(1)}(x) \frac{\partial \sigma}{\partial x}\left(x, X_{t}(x)\right) d W_{t} \\
& +X_{t}^{(1)}(x) \int_{-\infty}^{\infty} \frac{\partial g}{\partial x}\left(x, X_{t}(x), y\right)\left(N(d t, d y)-\nu_{t}(d y) d t\right) .
\end{aligned}
$$

We continue the proof by induction on $n \geq 1$. We assume that

$$
Z_{t}^{(n-1)}(x):=\left(X_{t}(x), \frac{\partial X_{t}}{\partial x}(x), \ldots, \frac{\partial^{n-1} X_{t}}{\partial x^{n-1}}(x)\right)^{\top} \in \mathbb{R}^{n}
$$


is solution of the $n$-dimensional SDE

$$
\begin{aligned}
Z_{t}^{(n-1)}(x)= & z^{(n-1)}+\int_{0}^{t} r^{(n-1)}\left(s, Z_{s}^{(n-1)}\right) d s+\int_{0}^{t} \sigma^{(n-1)}\left(s, Z_{s}^{(n-1)}\right) d W_{s} \\
& +\int_{0}^{t} \int_{-\infty}^{\infty} g^{(n-1)}\left(t, Z_{s^{-}}^{(n-1)}(x), y\right)\left(N(d s, d y)-\nu_{s}(d y) d s\right)
\end{aligned}
$$

with

$$
z_{0}^{(n-1)}=x, \quad z_{1}^{(n-1)}=1 \text { and } z_{k}^{(n-1)}=0, \quad k \geq 2
$$

and

a) $r^{(n-1)}:[0, T] \times \mathbb{R}^{n} \rightarrow \mathbb{R}^{n}$ is given by

$$
r^{(n-1)}\left(t, z^{(n-1)}\right)=\left(r_{0}^{(n-1)}, \ldots, r_{n-1}^{(n-1)}\right)^{\top}=\left(r^{(n-2)}\left(t, z^{(n-2)}\right), r_{n-1}^{(n-1)}\left(t, z^{(n-1))}\right)^{\top}\right.
$$

where

$$
r_{n-1}^{(n-1)}\left(s, z^{(n-1)}\right)=\sum_{\pi \in \Pi[n-1]} \frac{\partial^{|\pi|} r}{\partial x^{|\pi|}}\left(s, z^{(n-1)}\right) \prod_{B \in \pi} z_{|B|}^{(n-1)},
$$

b) $\sigma^{(n-1)}:[0, T] \times \mathbb{R}^{n} \rightarrow \mathbb{R}^{n}$ is given by

$$
\sigma^{(n-1)}\left(t, z^{(n-1)}\right)=\left(\sigma_{0}^{(n-1)}, \ldots, \sigma_{n-1}^{(n-1)}\right)^{\top}=\left(\sigma^{(n-2)}\left(t, z^{(n-2)}\right), \sigma_{n-1}^{(n-1)}\left(t, z^{(n-1)}\right)\right)^{\top}
$$

where

$$
\sigma_{n-1}^{(n-1)}\left(s, z^{(n-1)}\right)=\sum_{\pi \in \Pi[n-1]} \frac{\partial^{|\pi|} \sigma}{\partial x^{|\pi|}}\left(s, z^{(n-1)}\right) \prod_{B \in \pi} z_{|B|}^{(n-1)}
$$

c) $g^{(n-1)}:[0, T] \times \mathbb{R}^{n} \times \mathbb{R} \rightarrow \mathbb{R}^{n}$ is given by

$$
g^{(n-1)}\left(t, z^{(n-1)}, y\right)=\left(g_{0}^{(n-1)}, \ldots, g_{n-1}^{(n-1)}\right)^{\top}=\left(g^{(n-2)}\left(t, z^{(n-2)}, y\right), g_{n-1}^{(n-1)}\left(t, z^{(n-1)}, y\right)\right)^{\top}
$$

where

$$
g_{n-1}^{(n-1)}\left(s, z^{(n-1)}, y\right)=\sum_{\pi \in \Pi[n-1]} \frac{\partial^{|\pi|} g}{\partial x^{|\pi|}}\left(s, z^{(n-1)}, y\right) \prod_{B \in \pi} z_{|B|}^{(n-1)}
$$

where $\Pi[n-1]$ stands for the set of partition of $\{1, \ldots, n-1\}$. We also assume that $X_{t}^{(k)}(x)$ is solution of the $\operatorname{SDE}(4.1)$ for $k=0, \ldots, n-1$. 
Observe first that (4.3) holds for $Z^{(0)}=X(x)$ since in this case (4.3) reduces to (1.1) with

$$
r^{(0)}\left(t, z^{(0)}\right)=r\left(t, z^{(0)}\right), \quad \sigma^{(0)}\left(t, z^{(0)}\right)=\sigma\left(t, z^{(0)}\right), \quad g^{(0)}\left(t, z^{(0)}, y\right)=g\left(t, z^{(0)}, y\right) .
$$

Next, regarding $Z^{(1)}$, by (1.1) and (4.2) we have

$$
\begin{aligned}
Z^{(1)}(t)= & \left(\begin{array}{l}
X_{t} \\
X_{t}^{(1)}
\end{array}\right)=\left(\begin{array}{c}
1 \\
0
\end{array}\right)+\int_{0}^{t}\left(\begin{array}{c}
r\left(s, X_{s}\right) \\
\frac{\partial r}{\partial x}\left(s, X_{s}\right) X_{t}^{(1)}
\end{array}\right) d s+\int_{0}^{t}\left(\begin{array}{c}
\sigma\left(s, X_{s}\right) \\
\frac{\partial \sigma}{\partial x}\left(s, X_{s}\right) X_{s}^{(1)}
\end{array}\right) d W_{s} \\
& +\int_{0}^{t} \int_{-\infty}^{\infty}\left(\begin{array}{c}
g\left(s, X_{s^{-}}, y\right) \\
g\left(s, X_{s^{-}}, y\right) X_{s^{-}}^{(1)}
\end{array}\right)\left(N(d s, d y)-\nu_{s}(d y) d s\right)
\end{aligned}
$$

which is $(4.3)$ for $Z^{(1)}$ with

$$
\begin{aligned}
r^{(1)}\left(t, z^{(1)}\right) & =\left(\begin{array}{c}
r\left(t, z_{0}^{(1)}\right) \\
\frac{\partial r}{\partial x}\left(t, z_{0}^{(1)}\right) z_{1}^{(1)}
\end{array}\right), \quad \sigma^{(1)}\left(t, z^{(1)}\right)=\left(\begin{array}{c}
\sigma\left(t, z_{0}^{(1)}\right) \\
\frac{\partial \sigma}{\partial x}\left(t, z_{0}^{(1)}\right) z_{1}^{(1)}
\end{array}\right), \\
g^{(1)}\left(t, z^{(1)}, y\right) & =\left(\begin{array}{c}
g\left(t, z_{0}^{(1)}, y\right) \\
\frac{\partial g}{\partial x}\left(t, z_{0}^{(1)}, y\right) z_{1}^{(1)}
\end{array}\right),
\end{aligned}
$$

corresponding indeed to $(4.4)-(4.7)$ in this case. We now show that $Z^{(n)}(x)$ solves an SDE similar to (4.3), and that $X_{t}^{(n)}$ is solution to (4.1) for the index $n$. Since $Z^{(n-1)}(x)$ is solution to (4.3), by Theorem $6-29$ in [BGJ87], $\nabla_{z^{(n-1)}} Z^{(n-1)}$ is solution of the $M_{n, n}(\mathbb{R})$-valued matrix equation

$$
\begin{aligned}
& \nabla_{z^{(n-1)}} Z_{t}^{(n-1)}(x)=I_{n, n}+\int_{0}^{t} \nabla_{z^{(n-1)}} r^{(n-1)}\left(s, Z_{s}^{(n-1)}(x)\right) \nabla_{z^{(n-1)}} Z_{s}^{(n-1)}(x) d s \\
& \quad+\int_{0}^{t} \nabla_{z^{(n-1)}} \sigma^{(n-1)}\left(s, Z_{s}^{(n-1)}(x)\right) \nabla_{z^{(n-1)}} Z_{s}^{(n-1)}(x) d W_{s} \\
& \quad+\int_{0}^{t} \int_{-\infty}^{\infty} \nabla_{z^{(n-1)}} g^{(n-1)}\left(s, Z_{s^{-}}^{(n-1)}(x), y\right) \nabla_{z^{(n-1)}} Z_{s^{-}}^{(n-1)}(x)\left(N(d s, d y)-\nu_{s}(d y) d s\right) .
\end{aligned}
$$

With the notation $\nabla_{z^{(n-1)}}=\left(\frac{\partial}{\partial x}, \frac{\partial}{\partial z_{1}}, \ldots, \frac{\partial}{\partial z_{n-1}}\right)$, extracting the first column for the matrix equality in (4.8) we have

$$
\begin{aligned}
& \frac{\partial Z_{t}^{(n-1)}}{\partial x}(x)=(1,0, \ldots, 0)^{\top} \\
& +\int_{0}^{t} \nabla_{z^{(n-1)}} r^{(n-1)}\left(s, Z_{s}^{(n-1)}(x)\right) \frac{\partial Z_{s}^{(n-1)}}{\partial x}(x) d s+\int_{0}^{t} \nabla_{z^{(n-1)}} \sigma^{(n-1)}\left(s, Z_{s}^{(n-1)}(x)\right) \frac{\partial Z_{s}^{(n-1)}}{\partial x}(x) d W_{s}
\end{aligned}
$$




$$
+\int_{0}^{t} \int_{-\infty}^{\infty} \nabla_{z^{(n-1)}} g^{(n-1)}\left(s, Z_{s^{-}}^{(n-1)}(x), y\right) \frac{\partial Z_{s^{-}}^{(n-1)}}{\partial x}(x)\left(N(d s, d y)-\nu_{s}(d y) d s\right) .
$$

Next, for the leftmost entry in (4.9) we have

$$
\begin{aligned}
& \frac{\partial^{n} X_{t}}{\partial x^{n}}(x) \\
& =\int_{0}^{t}\left(\frac{\partial r_{n-1}^{(n-1)}}{\partial x}\left(s, Z_{s}^{(n-1)}(x)\right) \frac{\partial X_{s}}{\partial x}(x)+\sum_{i=1}^{n-1} \frac{\partial r_{n-1}^{(n-1)}}{\partial z_{i}^{(n-1)}}\left(s, Z_{s}^{(n-1)}(x)\right) \frac{\partial^{i+1} X_{s}}{\partial x^{i+1}}(x)\right) d s \\
& +\int_{0}^{t}\left(\frac{\partial \sigma_{n-1}^{(n-1)}}{\partial x}\left(s, Z_{s}^{(n-1)}(x)\right) \frac{\partial X_{s}}{\partial x}(x)+\sum_{i=1}^{n-1} \frac{\partial \sigma_{n-1}^{(n-1)}}{\partial z_{i}^{(n-1)}}\left(s, Z_{s}^{(n-1)}(x)\right) \frac{\partial^{i+1} X_{s}}{\partial x^{i+1}}(x)\right) d W_{s} \\
& +\int_{0}^{t} \int_{-\infty}^{\infty}\left(\frac{\partial g_{n-1}^{(n-1)}}{\partial x}\left(s, Z_{s^{-}}^{(n-1)}(x), y\right) \frac{\partial X_{s}}{\partial x}(x)+\sum_{i=1}^{n-1} \frac{\partial g_{n-1}^{(n-1)}}{\partial z_{i}^{(n-1)}}\left(s, Z_{s^{-}}^{(n-1)}(x), y\right) \frac{\partial^{i+1} X_{s}}{\partial x^{i+1}}(x)\right) \\
& \left(N(d s, d y)-\nu_{s}(d y) d s\right) .
\end{aligned}
$$

Putting together (4.3) and (4.10) yields an equation for $Z^{(n)}(x)$ similar to (4.3), and (4.10) proves (4.1) for $X^{(n)}(x)=\frac{\partial^{n}}{\partial x^{n}} X(x)$. Indeed, from (4.10) we recover the expressions of $r_{n}^{(n)}, \sigma_{n}^{(n)}$ and $g_{n}^{(n)}$ as in (4.5)-(4.7), which achieves the induction. For instance, using the expression (4.5) of $r_{n-1}^{(n-1)}$, we have

$$
\begin{aligned}
r_{n}^{(n)}\left(s, z^{(n)}\right)= & z_{1}^{(n)} \frac{\partial r_{n-1}^{(n-1)}}{\partial x}\left(s, z^{(n-1)}\right)+z_{i+1}^{(n)} \sum_{i=1}^{n-1} \frac{\partial r_{n-1}^{(n-1)}}{\partial z_{i}^{(n-1)}}\left(s, z^{(n-1)}\right) \\
= & z_{1}^{(n)} \frac{\partial}{\partial x}\left(\sum_{\pi \in \Pi[n-1]} \frac{\partial^{|\pi|} r}{\partial x^{|\pi|}}\left(s, z^{(n)}\right) \prod_{B \in \pi} z_{|B|}^{(n-1)}\right) \\
& +\sum_{i=1}^{n-1} z_{i+1}^{(n)} \frac{\partial}{\partial z_{i}^{(n-1)}}\left(\sum_{\pi \in \Pi[n-1]} \frac{\partial^{|\pi|} r}{\partial x^{|\pi|}}\left(s, z^{(n)}\right) \prod_{B \in \pi} z_{|B|}^{(n)}\right) \\
= & z_{1}^{(n)} \sum_{\pi \in \Pi[n-1]} \frac{\partial^{|\pi|+1} r}{\partial x^{|\pi|+1}}\left(s, z^{(n)}\right)\left(\prod_{B \in \pi} z_{|B|}^{(n-1)}\right) \\
& +\sum_{i=1}^{n-1} z_{i+1}^{(n)} \sum_{\pi \in \Pi[n-1]} \frac{\partial^{|\pi|} r}{\partial x^{|\pi|}}\left(s, z^{(n)}\right) \frac{\partial}{\partial z_{i}^{(n-1)}} \prod_{B \in \pi} z_{|B|}^{(n)} .
\end{aligned}
$$

In the second term above we have

$$
\frac{\partial}{\partial z_{i}^{(n-1)}} \prod_{B \in \pi} z_{|B|}^{(n)}=\frac{\partial}{\partial z_{i}^{(n-1)}} \prod_{j=1}^{n-1}\left(z_{j}^{(n)}\right)^{\#\{B \in \pi:|B|=j\}}
$$




$$
=\#\{B \in \pi:|B|=i\}\left(z_{i}^{(n)}\right)^{\#\{B \in \pi:|B|=i\}-1} \prod_{\substack{1 \leq j \leq n \\ j \neq i}}\left(z_{j}^{(n)}\right)^{\#\{B \in \pi:|B|=j\}} .
$$

We note that $\Pi[n]$ consists of partitions $\pi \in \Pi[n-1]$ with either the addition of $\{n\}$ as a new block, or the completion of an existing block by $\{n\}$. In the latter case, if $\{n\}$ is added to a block of size $j$ the new partition of $\Pi[n]$ obtained in this way will have one block of size $j$ less and one block of size $j+1$ more, and there are $\#\{B \in \pi:|B|=j\}$ such blocks. We conclude that the sums in (4.11) and (4.12) are effectively over $\Pi[n]$, which yields (4.5) for $r_{n}^{(n)}$, are as follows:

$$
r_{n}^{(n)}\left(s, z^{(n)}\right)=\sum_{\pi \in \Pi[n]} \frac{\partial^{|\pi|} r}{\partial x^{|\pi|}}\left(s, z^{(n-1)}\right) \prod_{B \in \pi} z_{|B|}^{(n-1)} .
$$

Similar computations yield also (4.6) and (4.7) and achieves the induction proving Proposition 4.1.

\section{$5 \quad$ Regularity of stochastic flows}

In this section we consider the solution $\left(X_{t}(x)\right)_{t \in[0, T]}$ of SDE (1.1), for which Proposition 4.1 gives condition for the differentiability of the flow $x \mapsto X_{t}(x)$ up to any order $n$. The next Theorem 5.1 deals with the integrability of order $q \geq 2$ of the flow derivatives, based on Corollaries 2.3 and 3.2, see also Theorem 3.3 in [Kun04] which only covers first order differentiability. We let $\|f(\cdot)\|_{\infty}$ denote the supremum of functions $f$ on $\mathbb{R}$.

Theorem 5.1 Let $n \geq 1$ and $q \geq 2$, and assume that (1.6) and $\left(A_{n}\right)$ hold. Then, for all $k=1, \ldots, n$ we have

$$
\sup _{x \in \mathbb{R}} \mathbb{E}\left[\sup _{t \in[0, T]}\left|X_{t}^{(k)}(x)\right|^{q}\right]<\infty .
$$

Proof. Since $X^{(n)}$ in (4.1) is expressed in terms of $X^{(k)}$ for $k<n$, deriving a moment of order $q$ for $X^{(n)}$ requires to show the existence of moments of order $q$ of certain products of the $X^{(k)}$. Accordingly, from the Hölder inequality, higher moments of every $X^{(k)}, k<n$, are required in our argument, see (5.5) below. By Proposition 4.1, 


$$
\begin{aligned}
& X_{t}^{(k)}(x):=\frac{\partial^{k} X_{t}}{\partial x^{k}}(x) \text { solves the SDE } \\
& d X_{t}^{(k)}(x)=\left(\sum_{\pi \in \Pi[k]} \frac{\partial^{|\pi|} r}{\partial x^{|\pi|}}\left(x, X_{t}(x)\right) \prod_{B \in \pi} X_{t}^{(|B|)}(x)\right) d t \\
&+\left(\sum_{\pi \in \Pi[k]} \frac{\partial^{|\pi|} \sigma}{\partial x^{|\pi|}}\left(x, X_{t}(x)\right) \prod_{B \in \pi} X_{t}^{(|B|)}(x)\right) d W_{t} \\
&+\int_{-\infty}^{\infty}\left(\sum_{\pi \in \Pi[k]} \frac{\partial^{|\pi|} g}{\partial x^{|\pi|}}\left(t, X_{t}(x), z\right) \prod_{B \in \pi} X_{t}^{(|B|)}(x)\right)\left(N(d t, d z)-\nu_{t}(d z) d t\right),
\end{aligned}
$$

with $X_{0}^{(k)}(x)=0$ for $k \geq 2$, where the sum over $\pi$ runs in the set $\Pi[k]$ of all partitions of $\{1,2, \ldots, k\}$. In order to prove $(5.1)$ for $k=1, \ldots, n$ we shall prove

$$
\sup _{x \in \mathbb{R}} \mathbb{E}\left[\sup _{t \in[0, T]}\left|X_{t}^{(k)}(x)\right|^{p_{k}}\right]<\infty
$$

by induction, for the order $p_{k}$ defined by

$$
p_{k}=q \frac{n !}{k !}
$$

For $k=1,(5.2)$ reduces to the affine equation

$$
d X_{t}^{(1)}(x)=a_{t}^{(1)} X_{t}^{(1)}(x) d t+b_{t}^{(1)} d W_{t}+X_{t^{-}}^{(1)}(x) \int_{-\infty}^{\infty} c_{t^{-}}^{(1)}(z)\left(N(d t, d z)-\nu_{t}(d z) d t\right)
$$

of the form (3.8), with $X_{0}^{(1)}(x)=1$ and

$$
a_{t}^{(1)}=\frac{\partial r}{\partial x}\left(t, X_{t}(x)\right), \quad b_{t}^{(1)}=\frac{\partial \sigma}{\partial x}\left(t, X_{t}(x)\right), \quad c_{t}^{(1)}(y)=\frac{\partial g}{\partial x}\left(t, X_{t}(x), y\right),
$$

see also Theorem 6-29 in [BGJ87]. Since $\left(a_{t}^{(1)}\right)_{t \in[0, T]},\left(b_{t}^{(1)}\right)_{t \in[0, T]} \in L^{p_{1}}\left([0, T], L^{\infty}(\Omega)\right)$ under $\left(A_{n}\right)$ and since Conditions (3.2)-(3.3) are satisfied by $c^{(1)}$ with $p=p_{1}=n ! q$ under (1.7), Corollary 3.2 shows that $X_{t}^{(1)}(x)$ admits a moment of order $p_{1}$, uniformly bounded in $t \in[0, T]$, that is (5.3) holds true for $k=1$.

Further, we assume that (5.3) holds true with order $p_{l}$ for $l=1, \ldots, k-1$ and we show that it remains true for the rank $l=k$. We note that (5.2) is thus an affine SDE of the form (3.8) in $X_{t}^{(k)}(x)$, with the random coefficients

$$
a_{x, t}^{(k)}:=\frac{\partial r}{\partial x}\left(t, X_{t}(x)\right)
$$




$$
\begin{aligned}
& b_{x, t}^{(k)}:=\frac{\partial \sigma}{\partial x}\left(t, X_{t}(x)\right), \\
& c_{x, t}^{(k)}(z):=\frac{\partial g}{\partial x}\left(t, X_{t}(x), z\right) \\
& u_{x, t}^{(k)}:=\sum_{\pi \in \Pi[k] \backslash\{1, \ldots, k\}} \frac{\partial^{|\pi|} r}{\partial x^{|\pi|}}\left(t, X_{t}(x)\right) \prod_{B \in \pi} X_{t}^{(|B|)}(x), \\
& v_{x, t}^{(k)}:=\sum_{\pi \in \Pi[k] \backslash\{1, \ldots, k\}} \frac{\partial^{|\pi|} \sigma}{\partial x^{|\pi|}}\left(t, X_{t}(x)\right) \prod_{B \in \pi} X_{t}^{(|B|)}(x), \\
& w_{x, t}^{(k)}(z):=\sum_{\pi \in \Pi[k] \backslash\{1, \ldots, k\}} \frac{\partial^{|\pi|} g}{\partial x^{|\pi|}}\left(t, X_{t}(x), z\right) \prod_{B \in \pi} X_{t}^{(|B|)}(x),
\end{aligned}
$$

as in (3.9). In order to show that $X_{t}^{(k)}(x)$ satisfies (5.3), we shall apply Corollary 3.2 for every $p_{k}$ as in (5.3) to the affine $\operatorname{SDE}(5.2)$ written as (3.12) and parameterized by the initial condition $x \in \mathbb{R}$, after checking that $\left(a_{x, t}^{(k)}\right)_{t \in[0, T]},\left(b_{x, t}^{(k)}\right)_{t \in[0, T]} \in$ $L^{p_{k}}\left([0, T], L^{\infty}(\Omega \times \mathbb{R})\right),\left(u_{x, t}^{(k)}\right)_{t \in[0, T]},\left(v_{x, t}^{(k)}\right)_{t \in[0, T]} \in L^{p_{k}}\left([0, T] \times \Omega, L^{\infty}(\mathbb{R})\right)$, and the conditions (3.10)-(3.11) for $\left(c_{x, t}^{(k)}(z)\right)_{t \in[0, T]},\left(w_{x, t}^{(k)}(z)\right)_{t \in[0, T]}$ hold, as follows.

i) The conditions

$$
\sup _{x \in \mathbb{R}} \int_{0}^{T}\left\|a_{x, t}^{(k)}\right\|_{\infty}^{p_{k}} d t<\infty, \quad \sup _{x \in \mathbb{R}} \int_{0}^{T}\left\|b_{x, t}^{(k)}\right\|_{\infty}^{p_{k}} d t<\infty,
$$

follow immediately from Assumption $\left(A_{n}\right)$ on $\partial r / \partial x$ and $\partial \sigma / \partial x$. On the other hand, regarding $c^{(k)}$, the bounds

$$
\int_{0}^{T}\left\|\int_{-\infty}^{\infty}\left|c_{x, t}^{(k)}(z)\right|^{2} \nu_{t}(d z)\right\|_{\infty}^{p_{k} / 2} d t<\infty \text { and } \int_{0}^{T}\left\|\int_{-\infty}^{\infty}\left|c_{x, t}^{(k)}(z)\right|^{p_{k}} \nu_{t}(d z)\right\|_{\infty} d t<\infty
$$

follow from (1.7) since $\sup _{x \in \mathbb{R}}\left\|c_{x, t}^{(k)}(y)\right\|_{\infty} \leq\left\|\frac{\partial g}{\partial x}(t, \cdot, y)\right\|_{\infty}$.

ii) We show that $\left(u_{x, t}^{(k)}\right)_{t \in[0, T]},\left(v_{x, t}^{(k)}\right)_{t \in[0, T]} \in L^{p_{k}}([0, T] \times \Omega)$, uniformly in $x \in \mathbb{R}$. Since the coefficients of (5.2) involve finite sums, we can deal with each summand separately using the convexity of $y \in \mathbb{R} \mapsto|y|^{p_{k}}$. For all $\pi \in \Pi[k] \backslash\{1, \ldots, k\}$, using $\left(A_{n}\right)$ and Hölder's inequality yields

$$
\mathbb{E}\left[\int_{0}^{T}\left|\frac{\partial^{|\pi|} r}{\partial x^{|\pi|}}\left(x, X_{t}(x)\right)\right|^{p_{k}} \prod_{B \in \pi}\left|X_{t}^{(|B|)}(x)\right|^{p_{k}} d t\right] \leq C \int_{0}^{T} \prod_{B \in \pi}\left(\mathbb{E}\left[\left|X_{t}^{(|B|)}(x)\right|^{p_{k}|\pi|}\right]\right)^{1 /|\pi|} d t .
$$


Since $\pi \in \Pi[k] \backslash\{1, \ldots, k\}$ has at least two blocks, for $B \in \pi$, we have $|B|<k$ and the induction hypothesis (5.3) applies for $X^{(|B|)}$. Additionally, $|\pi| \leq k$, and so

$$
p_{k}|\pi| \leq k p_{k}=p_{k-1} .
$$

As a consequence, by the induction hypothesis (5.1) applied to each $X_{t}^{(|B|)}(x)$, we have

$$
\sup _{x \in \mathbb{R}} \mathbb{E}\left[\sup _{t \in[0, T]}\left|X_{t}^{(|B|)}(x)\right|^{p_{k}|\pi|}\right]<\infty
$$

for each $B \in \pi$ and (5.5) ensures that

$$
\sup _{x \in \mathbb{R}} \mathbb{E}\left[\int_{0}^{T}\left|u_{x, t}^{(k)}\right|^{p_{k}} d t\right]<\infty,
$$

and similarly for $v_{x, t}^{(k)}$ we find:

$$
\sup _{x \in \mathbb{R}} \mathbb{E}\left[\int_{0}^{T}\left|v_{x, t}^{(k)}\right|^{p_{k}} d t\right]<\infty .
$$

iii) Verification of (3.2) for $w_{x, t}^{(k)}(z)$. Again, since the set $\Pi[k]$ of partitions of $\{1,2, \ldots, k\}$ is finite and $y \in \mathbb{R} \mapsto|y|^{2}, y \in \mathbb{R} \mapsto|y|^{p_{k} / 2}$ are both convex functions, we can deal with each summand separately. For all $\pi \in \Pi[k] \backslash\{1, \ldots, k\}$ we have

$$
\begin{aligned}
\mathbb{E} & {\left[\int_{0}^{T}\left(\int_{-\infty}^{\infty}\left|\frac{\partial^{|\pi|} g}{\partial x^{|\pi|}}\left(t, X_{t}(x), z\right)\right|^{2} \prod_{B \in \pi}\left|X_{t}^{(|B|)}(x)\right|^{2} \nu_{t}(d z)\right)^{p_{k} / 2} d t\right] } \\
& =\mathbb{E}\left[\int_{0}^{T}\left(\int_{-\infty}^{\infty}\left|\frac{\partial^{|\pi|} g}{\partial x^{|\pi|}}\left(t, X_{t}(x), z\right)\right|^{2} \nu_{t}(d z)\right)^{p_{k} / 2} \prod_{B \in \pi}\left|X_{t}^{(|B|)}(x)\right|^{p_{k}} d t\right] \\
& \leq \int_{0}^{T}\left(\int_{-\infty}^{\infty}\left\|\frac{\partial^{|\pi|} g}{\partial x^{|\pi|}}(t, \cdot, z)\right\|_{\infty}^{2} \nu_{t}(d z)\right)^{p_{k} / 2} \prod_{B \in \pi} \mathbb{E}\left[\left|X_{t}^{(|B|)}(x)\right|^{p_{k}|\pi|}\right]^{1 /|\pi|} d t \\
& \leq C \int_{0}^{T}\left(\int_{-\infty}^{\infty}\left\|\frac{\partial^{|\pi|} g}{\partial x^{|\pi|}}(t, \cdot, z)\right\|_{\infty}^{2} \nu_{t}(d z)\right)^{p_{k} / 2} d t,
\end{aligned}
$$

where the last bound uses (5.7) for the $X_{t}^{(|B|)}(x)$ with $|B|<k$ due to the induction hypothesis. This final bound is finite under (1.7), which ensures that

$$
\sup _{x \in \mathbb{R}} \mathbb{E}\left[\int_{0}^{T}\left(\int_{-\infty}^{\infty}\left|w_{x, t}^{(k)}(z)\right|^{2} \nu_{t}(d z)\right)^{p_{k} / 2} d t\right]<\infty .
$$


Similarly for (3.3), since $y \in \mathbb{R} \mapsto|y|^{2}$ and $y \in \mathbb{R} \mapsto|y|$ are both convex functions we have

$$
\begin{aligned}
\mathbb{E} & {\left[\int_{0}^{T} \int_{-\infty}^{\infty}\left|\frac{\partial^{|\pi|} g}{\partial x^{|\pi|}}\left(t, X_{t}(x), z\right)\right|^{p_{k}} \prod_{B \in \pi}\left|X_{t}^{(|B|)}(x)\right|^{p_{k}} \nu_{t}(d z) d t\right] } \\
& =\mathbb{E}\left[\int_{0}^{T} \int_{-\infty}^{\infty}\left|\frac{\partial^{|\pi|} g}{\partial x^{|\pi|}}\left(t, X_{t}(x), z\right)\right|^{p_{k}} \nu_{t}(d z) \prod_{B \in \pi}\left|X_{t}^{(|B|)}(x)\right|^{p_{k}} d t\right] \\
& \leq \int_{0}^{T} \int_{-\infty}^{\infty}\left\|\frac{\partial^{|\pi|} g}{\partial x^{|\pi|}}(t, \cdot, z)\right\|_{\infty}^{p_{k}} \nu_{t}(d z) \prod_{B \in \pi} \mathbb{E}\left[\left|X_{t}^{(|B|)}(x)\right|^{p_{k}|\pi|}\right]^{1 /|\pi|} d t \\
& \leq C \int_{0}^{T} \int_{-\infty}^{\infty}\left\|\frac{\partial^{|\pi|} g}{\partial x^{|\pi|}}(t, \cdot, z)\right\|_{\infty}^{p_{k}} \nu_{t}(d z) d t,
\end{aligned}
$$

using (5.7) for $X_{t}^{(|B|)}(x)$ with $|B|<n$, and we conclude to

$$
\sup _{x \in \mathbb{R}} \mathbb{E}\left[\int_{0}^{T} \int_{-\infty}^{\infty}\left|w_{x, t}^{(k)}(z)\right|^{p_{k}} \nu_{t}(d z) d t\right]<\infty .
$$

As a consequence, Theorem 3.1 can be applied to (5.2), which yields

$$
\sup _{t \in[0, T]} \mathbb{E}\left[\left|X_{t}^{(k)}(x)\right|^{p_{k}}\right]<\infty
$$

proving the induction hypothesis (5.3) for index $k$ and with order $p_{k}$ in (5.4). In particular, this proves Theorem 5.1.

Acknowledgement. We thank Yufei Zhang for corrections to Lemma 2.1 and Corollary 2.2 .

\section{References}

[App09] D. Applebaum. Lévy processes and stochastic calculus, volume 116 of Cambridge Studies in Advanced Mathematics. Cambridge University Press, Cambridge, second edition, 2009.

[BC86] R.F. Bass and M. Cranston. The Malliavin calculus for pure jump processes and applications to local time. Ann. Probab., 14(2):490-532, 1986.

[BGJ87] K. Bichteler, J.B. Gravereaux, and J. Jacod. Malliavin Calculus for Processes with Jumps, volume 2 of Stochastics Monographs. Gordon and Breach, 1987.

[Bic81] K. Bichteler. Stochastic integrators with stationary independent increments. Z. Wahrsch. Verw. Gebiete, 58(4):529-548, 1981.

[Bic02] K. Bichteler. Stochastic integration with jumps, volume 89 of Encyclopedia of Mathematics and its Applications. Cambridge University Press, Cambridge, 2002. 
[BJ83] K. Bichteler and J. Jacod. Calcul de Malliavin pour les diffusions avec sauts : existence d'une densité dans le cas unidimensionnel (in french) [malliavin calculus for diffusions with jumps: existence of a density in the one-dimensional case]. In Séminaire de Probabilités, XVII, volume 986 of Lecture Notes in Math., pages 132-157. Springer, Berlin, 1983.

[BP20] J.-C. Breton and N. Privault. Wasserstein distance estimates for jump-diffusion processes. Preprint, 22 pages, 2020.

[Hau11] E. Hausenblas. Maximal inequalities of the Itô integral with respect to Poisson random measures or Lévy processes on Banach spaces. Potential Anal., 35:223-251, 2011.

[HK05] D.J. Higham and P.E. Kloeden. Numerical methods for nonlinear stochastic differential equations with jumps. Numer. Math., 101(1):101-119, 2005.

[Kun84] H. Kunita. Stochastic differential equations and stochastic flows of diffeomorphisms. In École d'été de probabilités de Saint-Flour, XII-1982, volume 1097 of Lecture Notes in Math., pages 143-303. Springer, Berlin, 1984.

[Kun90] H. Kunita. Stochastic flows and stochastic differential equations, volume 24 of Cambridge Studies in Advanced Mathematics. Cambridge University Press, Cambridge, 1990.

[Kun04] H. Kunita. Stochastic differential equations based on Lévy processes and stochastic flows of diffeomorphisms. In Real and stochastic analysis, Trends Math., pages 305-373. Birkhäuser Boston, Boston, MA, 2004.

[LLP80] E. Lenglart, D. Lépingle, and M. Pratelli. Présentation unifiée de certaines inégalités de la théorie des martingales. In Seminar on Probability, XIV (Paris, 1978/1979) (French), volume 784 of Lecture Notes in Math., pages 26-52. Springer, Berlin, 1980.

[Pra83] M. Pratelli. Majorations dans $L^{p}$ du type Métivier-Pellaumail pour les semimartingales. In Seminar on probability, XVII, volume 986 of Lecture Notes in Math., pages 125-131. Springer, Berlin, 1983.

[Pro04] P. Protter. Stochastic integration and differential equations, volume 21 of Stochastic Modelling and Applied Probability. Springer-Verlag, Berlin, second edition, 2004.

[PT97] P. Protter and D. Talay. The Euler scheme for Lévy driven stochastic differential equations. Ann. Probab., 25(1):393-423, 1997.

[PW19] N. Privault and L. Wang. Stochastic SIR Lévy jump model with infinite activity. Preprint arXiv:1911.12924, 28 pages, 2019.

[Sit05] R. Situ. Theory of stochastic differential equations with jumps and applications. Mathematical and Analytical Techniques with Applications to Engineering. Springer, New York, 2005.

[ZWL15] X. Zhang, K. Wang, and D. Li. Stochastic periodic solutions of stochastic differential equations driven by Lévy process. Journal of Mathematical Analysis and Applications, 430:231-242, 2015.

[ZZ16] Y. Zhou and W. Zhang. Threshold of a stochastic SIR epidemic model with Lévy jumps. Phys. A, 446:204-216, 2016. 